\title{
Reflections for global public health research and evidence-based medicine during the COVID-19 pandemic
}

Healthcare professionals are often overburdened, with limited time to examine all current, best available evidence to inform their practice and clinical decision-making. This has been very much the case during the COVID-19 pandemic, with healthcare professionals at the frontline facing physical exhaustion, mental health challenges associated with stigma, limited family time, and the passing of those seeking healthcare. ${ }^{[1]}$ In addition to some of the psychosocial factors impacting on healthcare professionals, the surge in the available evidence during the COVID-19 pandemic made clinical decisionmaking cumbersome, as evidence-based medicine attempted to address clinical decision-making through the best available evidence together with clinical expertise and patient values. ${ }^{[2]}$

As the World Health Organization (WHO) declared a global public health emergency, the world saw an increase in the number of COVID-19 cases. Researchers and academics rapidly responded to this public health emergency through the generation of knowledge that aimed to be both relevant and timeous to address the needs of the pandemic and halt the number of infections for those at great risk. The rapid response meant that while researchers aimed to inform the public and the scientific community about advances in science, methodological and ethical dilemmas emerged as part of evidencebased decision-making. Cutting corners and producing evidence rapidly often meant that researchers and academics needed thoughtful reflection about whether they are guilty of exploiting the COVID-19 pandemic for academic progression while losing methodological and ethical integrity. In April 2020, the WHO database reported 5362 COVID-19-related studies and numerous online COVID-19 collections (such as the Living Overview of Evidence platform and the Living Systematic Map of Evidence, to name but two). ${ }^{[3]}$ Would the adaptations to evidence-based medicine that the pandemic brought about change how evidence generation and synthesis is conducted in the future? Yes, this has already occurred, and will most certainly continue to do so. An example would be the move away from the traditional qualitative evidence generation, which often requires in-depth, in-person interaction, towards the more virtual space through online data collection methodological approaches to still yield data that are rich and detailed. Simultaneously, it could also suggest that this is the shift from traditional research methods and approaches to ones that are changing with the times - in light of the fourth industrial revolution and the current pandemic.

Yet, while there are many changes and adaptations to our traditional views of evidence-based medicine, generation and synthesis, we need to make sure that as researchers and academics we do not lose our methodological and ethical integrity. Some reflections that researchers and academics might need to consider to avoid jeopardising the methodological and ethical integrity of scientific contributions are set out in Fig. 1.

All attempts at global public health research and evidence-based medicine should take place while the researcher reflects on how she/ he can ensure that the knowledge generated and shared can be relevant to help clinicians and the general public, locally. The language that is used in the dissemination and knowledge translation of evidence that emerges from research during the COVID-19 pandemic should also consider the availability of evidence in languages other than English, both languages widely spoken internationally (such as the inclusion

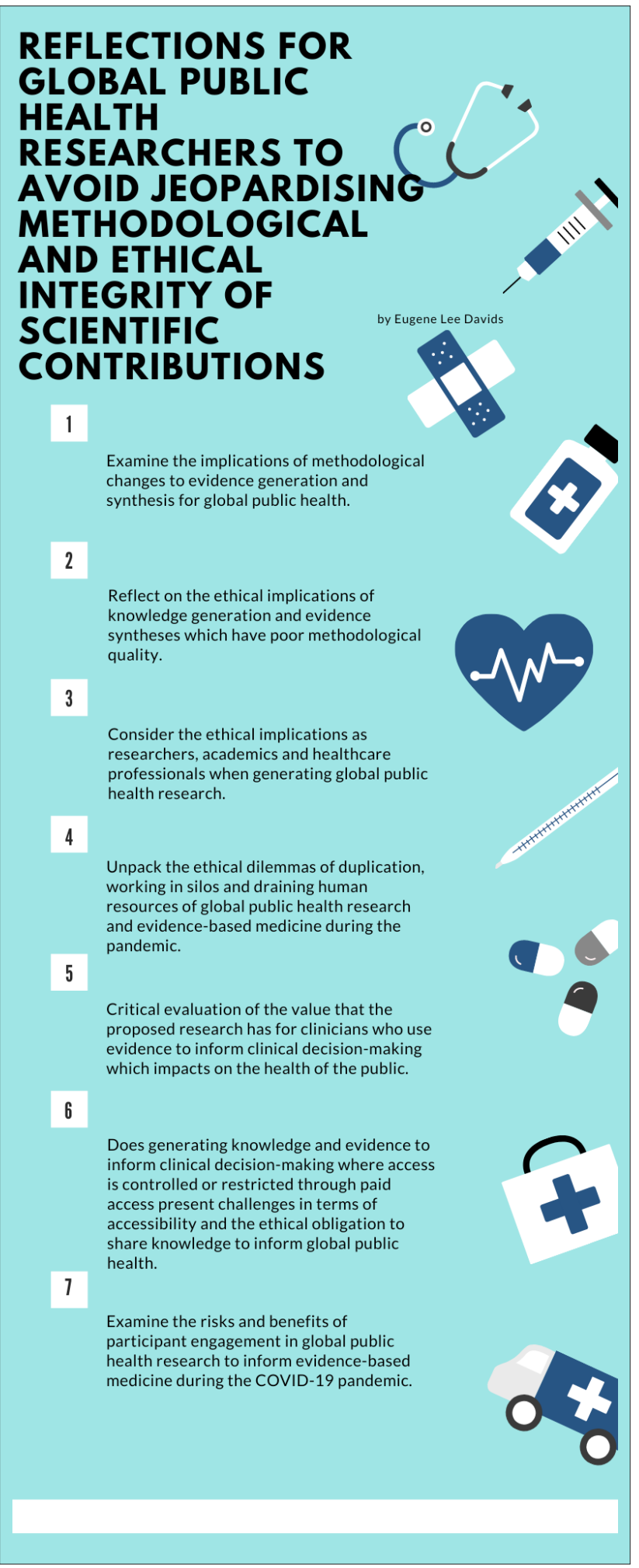

Fig. 1. Reflections to avoid jeopardising methodological and ethical integrity of scientific contributions during the COVID-19 pandemic. 
of Mandarin and Spanish) and locally (such as isiZulu, isiXhosa and Afrikaans). Researchers should reflect on how the use of only English in the dissemination of knowledge may have limited the overall aim of sharing knowledge.

\section{E L Davids}

Cochrane South Africa, South African Medical Research Council, South Africa; and Adolescent Health Research Unit, Division of Child and Adolescent Psychiatry, Department of Psychiatry and Mental Health, Faculty of Health Sciences, University of Cape Town, South Africa eugene.davids@mrc.ac.za
1. Chersich MF, Gray G, Fairlie L, et al. COVID-19 in Africa: Care and protection for frontline healthcare workers. Global Health 2020;16:46. https://doi.org/10.1186/s12992-020-00574-3

2. Akobeng AK. Principles of evidence-based medicine. Arch Dis Child 2005;90(8):837-840. https://doi. org/10.1136/adc.2005.071761

3. Ruano J, Gomez-Garcia F, Pieper D, Puljak L. What evidence-based medicine researchers can do to help clinicians fighting COVID-19? J Clin Epidemiol 2020;124:183-185. https://doi.org/10.1016/j. jclinepi.2020.04.015

S Afr Med J 2020;110(11):1070-1071. https://doi.org/10.7196/SAMJ.2020.v110i11.15276 